\title{
What's My Line? Glass Versus Paper for Cold Reading in Duologues
}

Jamie A. Ward

German Research Center for

Artificial Intelligence (DFKI)

Kaiserslautern, Germany

jamie@jamieward.net
Paul Lukowicz

German Research Center for

Artificial Intelligence (DFKI)

Kaiserslautern, Germany

paul.lukowicz@dfki.de

\begin{abstract}
Part of an actor's job is being able to cold read: to take words directly from the page and to read them as if they were his or her own, often without the chance to read the lines beforehand. This is particularly difficult when two or more actors need to perform a dialogue cold. The need to hold a paper script in hand hinders the actor's ability to move freely. It also introduces a visual distraction between actors trying to engage with one another in a scene. This preliminary study uses Google Glass displayed cue cards as an alternative to traditional scripts, and compares the two approaches through a series of two-person, cold-read performances. Each performance was judged by a panel of theatre experts. The study finds that Glass has the potential to aid performance by freeing actors to better engage with one another. However, it also found that by limiting the display to one line of script at a time, the Glass application used here makes it difficult for some actors to grasp the text. In a further study, when asked to later perform the text from memory, actors who had used Glass recalled only slightly fewer lines than when they had learned using paper.
\end{abstract}

\section{Author Keywords}

Eyeware; Glass; Applications; Theatre; Wearables

\section{ACM Classification Keywords}

I.2.m [Artificial Intelligence]: Miscellaneous 

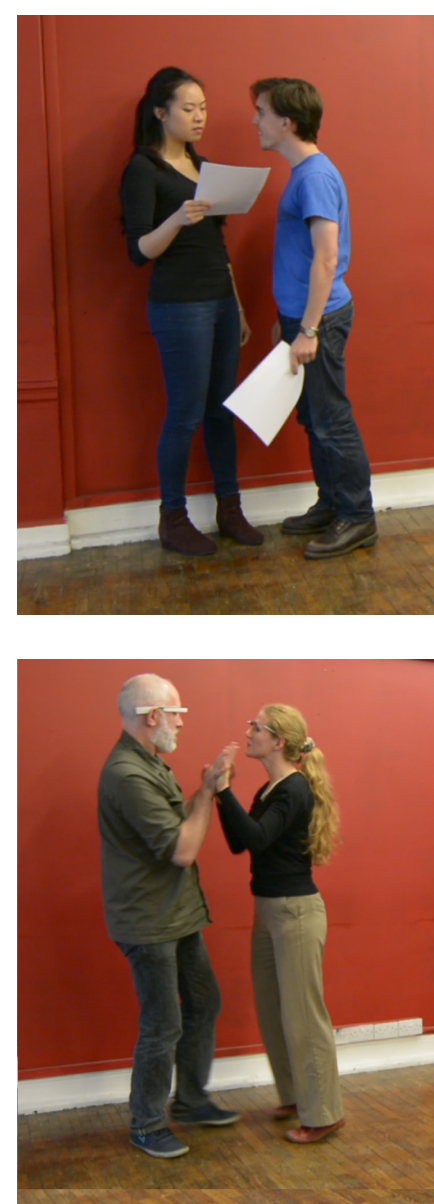

Figure 1: Actors performing duologues using Paper (top) and Glass (bottom)

\section{Introduction}

An actor's goal is to tell a story. Good or bad performances rest on how well actors speak, move, and interact with one another. In many instances, for example in audition, rehearsal, or during rehearsed readings, actors must perform a text cold. The challenge is to engage in the main task - telling the story and interacting with the other actor(s) without being distracted by the necessity of reading lines off a page. Cue cards, and scrolls, have long been used to help actors speak their lines [1], and the Autocue is widely used by presenters on TV. But these all require the speaker to either hold a card, or to frequently direct their gaze to a fixed point.

The peripheral vision, "eye-wear" computer, Google Glass, can convey information with minimal distraction, even during attention critical tasks such as driving [4]. It has also been used by musicians to display score, allowing navigation by wink and touch gesture [5]. Using a similar headmounted display (HMD) Okada et al. developed a contextaware prompter to aid presenters giving a speech [3]. That system used speech and sound recognition, combined with direct input from the user, to determine when to scroll to the next part of the script. The current work draws on that idea but introduces the additional complexity of two actors using Glass to help them perform a duologue (2 person scene). This paper describes a preliminary study on actors using Glass to cold read a script line-by-line versus using traditional paper scripts.

Actors in pairs worked on two short scenes in front of a panel of three experienced theatre directors. One scene is performed cold using paper scripts. In the other scene actors are prompted using Glass (see Figure 1). The directors evaluate each actor's performance according to two criteria: 1) how well they interact with one another - are they listening, and reacting, to what their partner says and does? And 2) how well they read the text - do they seem to understand what it is that they are saying? Broadly, the first of these (subjective) measures indicates engagement with the main task (of performance), the second indicates comprehension of the material. In a third analysis, actors are asked to put aside all script aids and attempt to re-perform the scenes from memory. Line recall rates are calculated and compared for learning using paper versus Glass.

\section{Experiment}

Ten classically-trained, professional actors, aged between 24 and 61 years old, were divided into five male(M)-female(F) pairings (M1\&F1,... M5\&F5). Two scenes from Shakespeare were chosen ${ }^{1}$. All actors were broadly familiar with these plays but none knew the lines of these characters in advance. Both scenes are around 40 lines of verse, split evenly between two characters, of similar complexity, and include opportunities for physical action.

Two Glass devices were set up to display a single line of verse at a time on each screen (Glassware 'card'). While one actor's line is displayed, as in Figure 2, the other actor gets a blank card. Each new line appears when an assistant presses a button on a smartphone linked via Bluetooth to each Glass. (This Wizard-of-Oz approach has the obvious drawback of introducing latency through both the assistant's and the wireless link's response times. For this reason timing is not analyzed in this initial study.)

Shortly before the study actors were given 10 minutes to familiarise themselves with the technology. Each couple had two sessions: one using paper (with either script $A$ or B), and one using Glass (with the other script). Before each session, couples were given 10 minutes to read and

\footnotetext{
${ }^{1}(A)$ Taming of the Shrew, 2.1 \& (B) Richard III, 1.2
} 


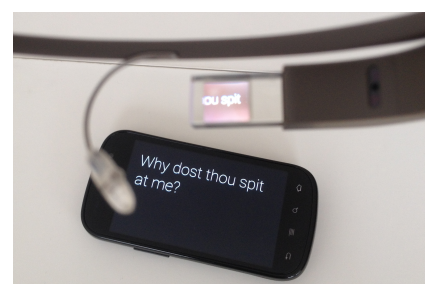

Figure 2: A line of text shown on a smartphone in the same format as it appears on Glass prepare using a (paper) copy of the text. Each session consisted of actors performing the scene three times: 1 st time cold, 2nd time following advice from a director, and 3rd time attempting the scene from memory. The directors judged each actor during the first two attempts using using a 5point Likert-scale, ('very bad' to 'very good'). These judgements are summarised in Figure 3.

The performances from memory are not assessed by the directors. Instead each time an actor forgets a line, an assistant with the script gives a prompt and the scene continues. Line recall rate (remembered lines $\div$ total lines) is charted for each actor and learning method in Figure 4.

\section{Discussion of Results}

Reaction to Scene Partner Directors judged actor engagement (interaction, listening and reacting to partner) on average slightly better when using Glass than when using paper scripts. Fewer reads were rated as having 'poor' reactions with Glass ( $8 \%$ ) versus paper (10\%). All directors (and many actors) commented that Glass forced actors to listen to their partner speaking, whereas paper would tempt actors to get distracted by the text ahead. The difference is slight, however, a fact that is partly explained by the actor questionnaires: firstly, actors are more familiar with paper and are well-trained in cold reading; and secondly, many actors, particularly those left-eye dominant, had difficulties in switching focus between their partner and the right-eye displayed text.

Engagement with Text The directors judged actors using Glass to be poorer at grasping the text (and conveying its meaning) than those using paper. One clue to this can be found in the comments, e.g. F5: "I couldn't see line breaks - nor have an idea how long my thoughts might be", and M1 complained that he "Tended to wait for next words to

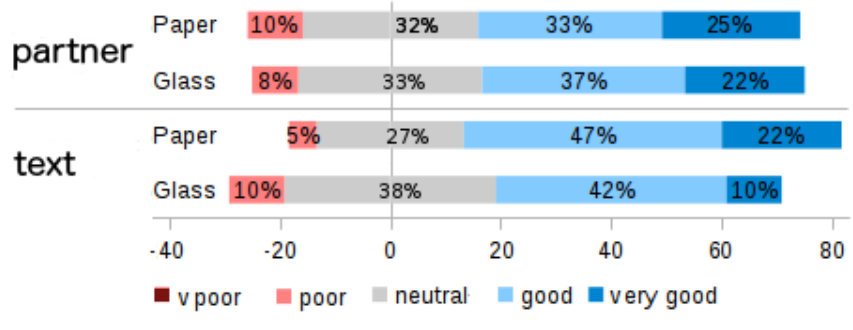

Figure 3: Frequency (over 40 runs) of the 3 directors' responses to each evaluation question: engagement with scene partner \& grasp of the text

appear more than use the thought". The underlying intention, or thought, behind each spoken line of verse can often continue over several lines and is critical to how an actor interprets and speaks a text. Actors felt limited by Glass only displaying one line at a time.

Line Learning The average line recall rates shown in Figure 4 are slightly higher for paper $(\mathrm{M}=.51, \mathrm{SD}=.20)$ than Glass $(M=.44, S D=.15)$, though this is not significant. Some actors responded that they found recall easier having used paper because they could remember the text using visual memory. This supports long-established findings on the importance of visual page cues to learning [2]. Four actors reported that learning was easier using Glass. F3 thought her learning was helped by not having to search for words on a page: "you can grab which bit it is and go".

Overall Impressions "Actors looked freer to move with Glass", wrote one director. The actors were split evenly between those who found themselves physically freer using Glass, and those who struggled with it. One participant, F5, who has an impairment with her right hand, was enthusias- 


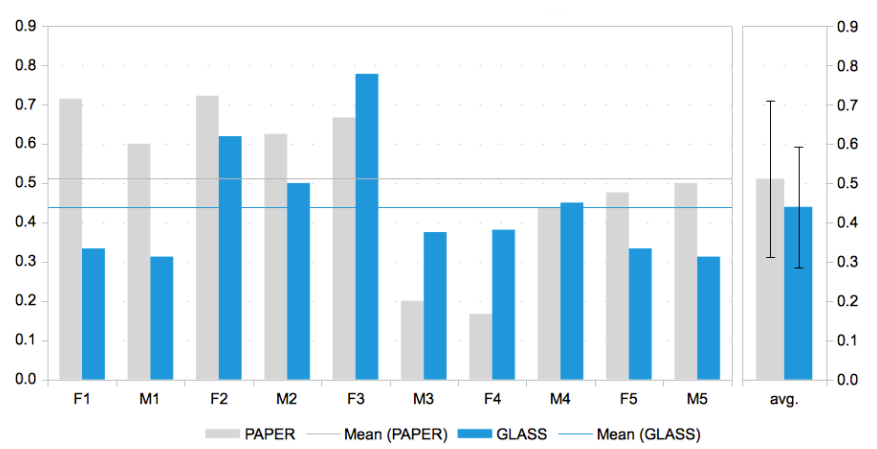

Figure 4: Line recall rates for each actor, and the average (with standard deviation), having learned using paper v. Glass

tic about the technology. "I really struggle holding paper... My left hand [is] completely blocked which is also my more expressive", she wrote. However she struggled using Glass because of an astigmatism in her right eye which caused her contact lens to slip.

Conclusion and Future Work Glass could be a feasible replacement to paper scripts for actors doing a cold read. Most of the actors in this study benefited from some physical freedom using Glass, though some struggled with the right-side only design, and some with the limitation of displaying one line at a time. A future iteration of this work will be to explore different ways of presenting script information so that actors get a sense of what is to come after each line. Part of this will be to try alternative HMDs. The ultimate goal of this research is to replace the "Wizard" with a system that automatically recognises actor-to-actor interaction (through voice, movement, and eye gaze) and use this information to completely automate prompting.

\section{REFERENCES}

1. Andy Kirtland. 2015. An Unrehearsed Cue Script Perspective on Love's Labour's Lost. Actes des congrés de la Société francaise Shakespeare 32 (2015). http://shakespeare.revues.org/3232

2. Eugene A Lovelace and Stephen D Southall. 1983. Memory for words in prose and their locations on the page. Memory \& Cognition 11, 5 (1983), 429-434.

3. Tomonari Okada, Tetsuya Yamamoto, Tsutomu Terada, and Masahiko Tsukamoto. 2011. Wearable MC System a System for Supporting MC Performances Using Wearable Computing Technologies. In Proceedings of the 2Nd Augmented Human International Conference (AH '11). ACM, New York, NY, USA, Article 25, 7 pages. DOI :

http://dx.doi.org/10.1145/1959826.1959851

4. Kathryn G Tippey, Elayaraj Sivaraj, Wil-Johneen Ardoin, Trey Roady, and Thomas K Ferris. 2014. Texting while driving using Google Glass Investigating the combined effect of heads-up display and hands-free input on driving safety and performance. In Proceedings of the Human Factors and Ergonomics Society Annual Meeting, Vol. 58. SAGE Publications, 2023-2027.

5. Cynthia Turner and Tyler Ehrlich. 2013-2014. Score Viewer. (2013-2014). http://glass.music.uga.edu/ 